\title{
Lattices and dimensional representations: matrix decompositions and ordering structures
}

\author{
Philippa E. Pattison ${ }^{\mathrm{a}, *}$, Ronald L. Breiger ${ }^{\mathrm{b}}$ \\ a Department of Psychology, University of Melbourne, Parkville, Vic. 3010, Australia \\ ${ }^{\mathrm{b}}$ Department of Sociology, University of Arizona, Tucson, AZ, USA
}

\begin{abstract}
This paper is concerned with some methods that attempt to provide simultaneous representation of dual relationships, such as ties of membership that connect persons and groups, or connections between organizations and agendas. We focus on vector space and lattice representations, and on techniques for simplifying the structure of dual networks. We demonstrate some fundamental similarities among these approaches.
\end{abstract}

(C) 2002 Elsevier Science B.V. All rights reserved.

Keywords: Matrix decomposition; Lattice representation; Boolean vector

\section{Introduction}

It has been recognized for many years that some fundamental dualities among social entities of different types can be understood by analyzing observed relations among pairs of sets (Breiger, 1974; Freeman, 1980; Wilson, 1982; Fararo and Doreian, 1984; Freeman and White, 1993; White and Duquenne, 1996). For instance, a binary relation indicating the membership of a set of persons in a set of groups provides not only an explicit representation of the co-constitution of groups and persons, but also an implicit representation of relationships among persons (in terms of the sets of groups to which they belong) and of relationships among groups (in terms of their memberships (Breiger, 1974, 1990)).

With his characteristic incisiveness, Freeman has played an important role in showing how the analysis and re-representation of such dual relations can enhance our understanding of these explicit and implicit relationships. Focusing first on $Q$-analysis (e.g. Freeman, 1980) and subsequently on Galois lattices (Freeman, 1992, 1996; Freeman and White, 1993), Freeman has opened productive pathways from mathematics to substantive, theoretical, and methodological innovation in social network analysis.

\footnotetext{
* Corresponding author. Tel.: +61-3-8344-6371; fax: +61-3-9347-6618.

E-mail address: p.pattison@psych.unimelb.edu.au (P.E. Pattison).
} 
This paper includes a review of some methods that attempt to provide simultaneous representation of dual relationships. Our main aims in the paper are two-fold. First, we discuss in some detail the case of dual relationships expressed through an observed binary relation between two sets. In particular, we explicate the relationship between representations based on Boolean vector spaces and lattice representations, and we identify situations in which they are equivalent. Second, we argue that a variety of different vector space representations can be (and have been) considered for relational data between sets, with an appropriate form depending on the nature of the data and the assumptions that it arguably sustains. Further, a general vector space representational form provides a natural unifying framework for the analysis of relational data between two sets.

Well-known examples of the two kinds of approaches we seek to relate are correspondence analysis and Galois lattice (also known as formal concept lattice) analysis. Recently, there has been increased appreciation of how both techniques can be combined within the same research study to enhance the investigation of relations among dual entities (such as the important persons and their qualities as named by an anorexic patient (Wolff and Gabler, 1998)). And Galois lattice techniques can be used to provide interpretation for dimensional analyses such as those produced by log-multiplicative statistical models (as in an illustrative analysis of supreme court data in Breiger (2000, pp. 102-106), studying individual justices and issue-areas in which each votes in the majority). Our paper demonstrates foundations for the joint use of the two kinds of approaches.

We refer, for convenience, to relations among persons and groups, but as already noted many other pairs of entities may be analyzed in exactly the same way, some familiar examples being individuals and event attendance patterns (Foster and Seidman, 1984), records of academic institutions with which researchers have been affiliated (Freeman, 1980), organizations and the components of their political agendas (Mische and Pattison, 2000), and so on.

A binary relation can be described in terms of a person $\times$ group array, in which $P=$ $\{1,2, \ldots, a\}$ is a set of persons, $G=\{1,2, \ldots, d\}$ is a set of groups and $\boldsymbol{X}$ is an $a \times d$ array for which $X_{i j}=1$, if the person $i$ belongs to group $j$ and $X_{i j}=0$ otherwise. In general, we assume that the sets $P$ and $G$ are distinct, so that the array $\boldsymbol{X}$ can be regarded having two modes (Arabie et al., 1987). In some cases, the relation may be valued, in which case possible values of $X_{i j}$ are discrete and come from a finite set $A$, or they are continuous and come from an interval in the set of real numbers.

\section{Vector space and lattice representations}

A natural starting point for the representation of binary arrays is with those approaches that assume a Boolean algebra for the set $A$ of possible values. In this case, values of $X_{i j}$ are assumed to lie in the set $A=\{0,1\}$ and there are three operations,.$+^{\mathrm{c}}$, defined by: $0+0=0.1=1.0=0.0=0 ; 0+1=1+0=1+1=1.1=1 ; 0^{\mathrm{c}}=1 ;$ and $1^{\mathrm{c}}=0(A$ is therefore a two-element Boolean algebra (Birkhoff, 1967)).

\subsection{Boolean vector spaces and associated lattices}

The rows (or columns) of the binary array $\boldsymbol{X}$ give rise to a Boolean vector space, defined in the following paragraphs. 
Definition. Let $V_{n}$ denote the set of all vectors of dimension $n$ :

$$
\boldsymbol{v}=\left[\begin{array}{llll}
a_{1} & a_{2} & \cdots & a_{n}
\end{array}\right]
$$

with each $a_{i} \in A, i=1,2, \ldots, n$. The system $V_{n}$ together with the operation of componentwise addition:

$$
\left[\begin{array}{llll}
a_{1} & a_{2} & \cdots & a_{n}
\end{array}\right]+\left[\begin{array}{llll}
b_{1} & b_{2} & \cdots & b_{n}
\end{array}\right]=\left[\begin{array}{llll}
a_{1}+b_{1} & a_{2}+b_{2} & \cdots & a_{n}+b_{n}
\end{array}\right],
$$

defines the Boolean vector space of dimension $n$. A subset $V$ of $V_{n}$ is a subspace of $V_{n}$ if it contains the zero vector $\left[\begin{array}{llll}0 & 0 & \cdots & 0\end{array}\right]$ and it is closed under addition. The span of a subset $W$ of $V_{n}$ is the intersection of all subspaces containing $W$. The vector space $V^{n}$ is defined analogously, comprising Boolean column vectors of dimension $n$ rather than row vectors.

Definition. Let $\boldsymbol{X}$ be a binary relation on $P \times G$. The span of the set of rows of $\boldsymbol{X}$ is a subspace of $V_{d}$, termed the row space of $\boldsymbol{X}$. The columns of $\boldsymbol{X}$ span a subspace of $V_{a}$, termed the column space of $\boldsymbol{X}$. Indeed, both vector spaces are partially ordered sets under the operation of comparison of vectors:

$\left[\begin{array}{llll}a_{1} & a_{2} & \cdots & a_{d}\end{array}\right] \geq\left[\begin{array}{llll}b_{1} & b_{2} & \cdots & b_{d}\end{array}\right], \quad$ iff $a_{i} \geq b_{i} \quad$ for each $i=1,2, \ldots, d$.

Note that if $\left[\begin{array}{llll}a_{1} & a_{2} & \cdots & a_{d}\end{array}\right] \geq\left[\begin{array}{llll}b_{1} & b_{2} & \cdots & b_{d}\end{array}\right]$, we may also write $\left[\begin{array}{llll}b_{1} & b_{2} & \cdots\end{array}\right.$ $\left.b_{d}\right] \leq\left[\begin{array}{llll}a_{1} & a_{2} & \cdots & a_{d}\end{array}\right]$; here, we use the two expressions interchangebly. It may easily be shown that each vector space defines a lattice.

Definition. A lattice $L$ is a partially ordered set $L$, with a partial order $\geq$, in which every pair of elements $a$ and $b$ have a least upper bound, or join, denoted by $a \vee b$, and a greatest lower bound, or meet, denoted by $a \wedge b$. The join $a \vee b$ is the (unique) element $x$ satisfying $x \geq a$ and $x \geq b$, and if $y$ is any other element satisfying $y \geq a$ and $y \geq b$, then $y \geq x$; the meet $a \wedge b$ is the unique element $z$ satisfying $a \geq z$ and $b \geq z$, and if $w$ is any other element satisfying $a \geq w$ and $b \geq w$, then $z \geq w$.

A lattice is generated by the rows of the matrix $\boldsymbol{X}$ under the addition operation and will be denoted by $L_{\mathrm{R}}(\boldsymbol{X})$ and termed the row lattice of $\boldsymbol{X}$; the columns generate a column lattice $L_{\mathrm{C}}(\boldsymbol{X})$ which is anti-isomorphic to $L_{\mathrm{R}}(\boldsymbol{X})$. A lattice $L$ is anti-isomorphic or dual to a lattice $K$ if there is a one-to-one mapping $\phi$ from $L$ to $K$ with the property that: $a \geq b$ in $L$ if and only if $\phi(a) \leq \phi(b)$ in $K$. In both $L_{\mathrm{R}}(\boldsymbol{X})$ and $L_{\mathrm{C}}(\boldsymbol{X})$, the join of two vectors is equal to their Boolean sum, while their meet is the maximal vector in the lattice which is less than or equal to both of them. The row lattice $L_{R}(X)$ has also been termed the Zareckii lattice by Boyd (1990).

For instance, the binary matrix $\boldsymbol{X}$ of Table 1 generates the row lattice whose partial order diagram is displayed in Fig. 1. The elements of the lattice are the set of Boolean vectors comprising the rows of $\boldsymbol{X}$ and all possible vectors constructed from the rows by component-wise addition. In Fig. 1, which is termed a partial order, or Hasse diagram, an element $a$ is displayed earlier and directly connected to an element $b$ if $a$ covers $b$, that is if $a \geq b$ and if $z$ is an element satisfying $a \geq z \geq b$ then either $z=a$ or $z=b$. Table 2 reports the join and meet of each pair of elements in the lattice. 
Table 1

A rectangular binary matrix $\boldsymbol{X}$

\begin{tabular}{llllll}
\hline 0 & 0 & 0 & 0 & 0 & 0 \\
0 & 1 & 0 & 1 & 0 & 0 \\
0 & 0 & 0 & 0 & 1 & 1 \\
1 & 0 & 1 & 1 & 0 & 0 \\
1 & 1 & 0 & 1 & 0 & 0 \\
0 & 1 & 0 & 1 & 1 & 1 \\
\hline
\end{tabular}

Definition. Let $B$ be a subset of the vector space $V_{n}$. A vector $v \in V_{n}$ is dependent on $B$ if $v$ is contained in the span of $B$. A set $B$ of vectors is independent if $\boldsymbol{v}$ is not dependent on $B \backslash\{\boldsymbol{v}\}$, for each $\boldsymbol{v} \in B$. A basis of a subspace $W$ of $V_{n}$ is an independent set $B$ whose span is $W$.

It may readily be shown that the basis of a subspace of $V_{n}$ is unique (Kim, 1982). Thus, the vector spaces generated by the rows and columns of $\boldsymbol{X}$ each have a unique basis, termed, respectively, the row basis and column basis of $\boldsymbol{X}$. The number of vectors in each of these unique row and column bases are termed the row rank, $r=r(\boldsymbol{X})$ and the column rank, $c=c(\boldsymbol{X})$ of $\boldsymbol{X}$, respectively. The row and column basis vectors may be identified from the lattices $L_{\mathrm{R}}(\boldsymbol{X})$ and $L_{\mathrm{C}}(\boldsymbol{X})$.

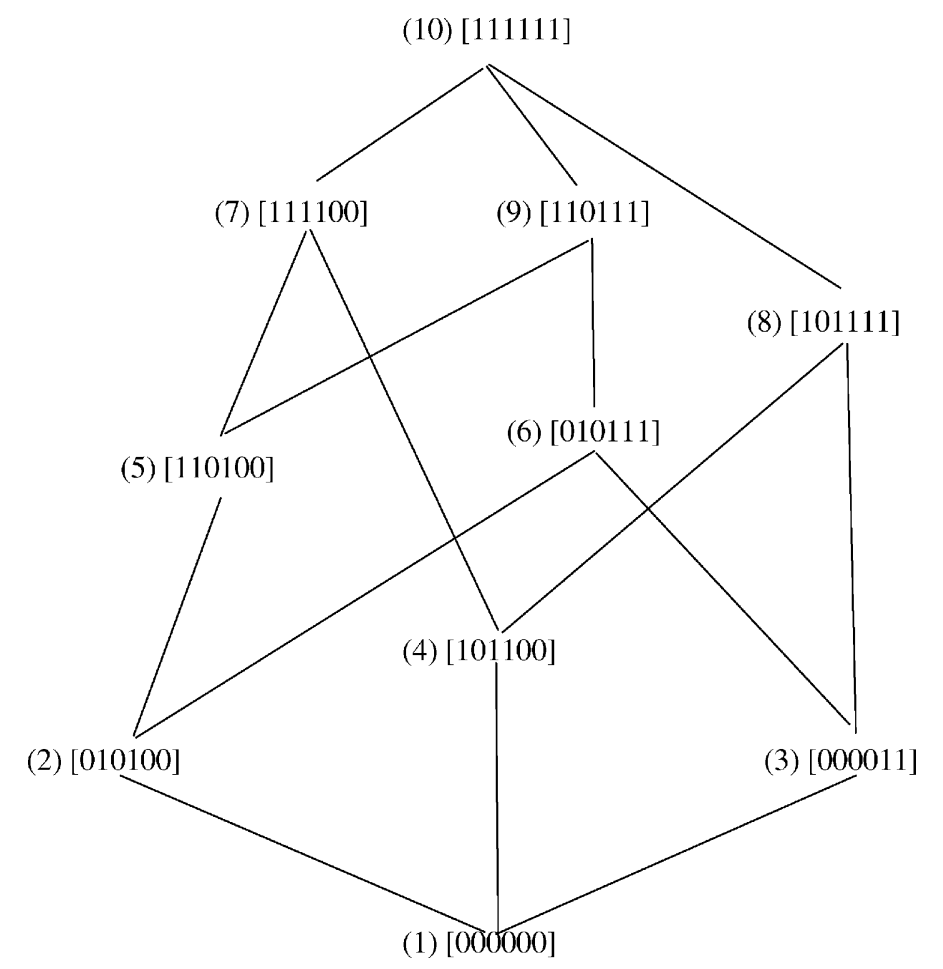

Fig. 1. The partial order diagram for the lattice of Table 1 (the vectors represent elements in the lattice $L_{\mathrm{R}}(\boldsymbol{X})$ ). 
Table 2

A lattice $L=L_{\mathrm{R}}(\boldsymbol{X})$ (the row lattice of the binary matrix $\boldsymbol{X}$ of Table 1)

\begin{tabular}{|c|c|c|c|c|c|c|c|c|c|c|}
\hline & 1 & 2 & 3 & 4 & 5 & 6 & 7 & 8 & 9 & 10 \\
\hline \multicolumn{11}{|c|}{ Meet table } \\
\hline 1 & 1 & 1 & 1 & 1 & 1 & 1 & 1 & 1 & 1 & 1 \\
\hline 2 & 1 & 2 & 1 & 1 & 2 & 2 & 2 & 1 & 2 & 2 \\
\hline 3 & 1 & 1 & 3 & 1 & 1 & 3 & 1 & 3 & 3 & 3 \\
\hline 4 & 1 & 1 & 1 & 4 & 1 & 1 & 4 & 4 & 1 & 4 \\
\hline 5 & 1 & 2 & 1 & 1 & 5 & 2 & 5 & 1 & 5 & 5 \\
\hline 6 & 1 & 2 & 3 & 1 & 2 & 6 & 2 & 3 & 6 & 6 \\
\hline 7 & 1 & 2 & 1 & 4 & 5 & 2 & 7 & 4 & 5 & 7 \\
\hline 8 & 1 & 1 & 3 & 4 & 1 & 3 & 4 & 8 & 3 & 8 \\
\hline 9 & 1 & 2 & 3 & 1 & 5 & 6 & 5 & 3 & 9 & 9 \\
\hline 10 & 1 & 2 & 3 & 4 & 5 & 6 & 7 & 8 & 9 & 10 \\
\hline \multicolumn{11}{|c|}{ Join table } \\
\hline 1 & 1 & 2 & 3 & 4 & 5 & 6 & 7 & 8 & 9 & 10 \\
\hline 2 & 2 & 2 & 6 & 7 & 5 & 6 & 7 & 10 & 9 & 10 \\
\hline 3 & 3 & 6 & 3 & 8 & 9 & 6 & 10 & 8 & 9 & 10 \\
\hline 4 & 4 & 7 & 8 & 4 & 7 & 10 & 7 & 8 & 10 & 10 \\
\hline 5 & 5 & 5 & 9 & 7 & 5 & 9 & 7 & 8 & 9 & 10 \\
\hline 6 & 6 & 6 & 6 & 10 & 9 & 6 & 10 & 10 & 9 & 10 \\
\hline 7 & 7 & 7 & 10 & 7 & 7 & 10 & 7 & 10 & 10 & 10 \\
\hline 8 & 8 & 10 & 8 & 8 & 10 & 10 & 10 & 8 & 10 & 10 \\
\hline 9 & 9 & 9 & 9 & 10 & 9 & 9 & 10 & 10 & 9 & 10 \\
\hline 10 & 10 & 10 & 10 & 10 & 10 & 10 & 10 & 10 & 10 & 10 \\
\hline
\end{tabular}

Definition. An element $x$ of a lattice $L$ is join-irreducible if $x \neq x_{\min }$ and $x=x_{1} \vee x_{2}$ implies $x=x_{1}$ or $x=x_{2}$.

Proposition 1. The row and column basis vectors of $\boldsymbol{X}$ are precisely the join-irreducible elements of $L_{\mathrm{R}}(\boldsymbol{X})$ and $L_{\mathrm{C}}(\boldsymbol{X})$, respectively.

Proof. For instance, see Kim (1982)

For example, the join-irreducible elements of the lattice of Fig. 1 are those labeled (2), (3), (4), and (5); hence, the row basis of the binary matrix $\boldsymbol{X}$ of Table 1 is:

$$
\begin{array}{rllllll}
\boldsymbol{b}_{1} & =\left[\begin{array}{llllll}
0 & 1 & 0 & 1 & 0 & 0
\end{array}\right], & \boldsymbol{b}_{2} & =\left[\begin{array}{llllll}
0 & 0 & 0 & 0 & 1 & 1
\end{array}\right], \\
\boldsymbol{b}_{3} & =\left[\begin{array}{llllll}
1 & 0 & 1 & 1 & 0 & 0
\end{array}\right], & \boldsymbol{b}_{4}=\left[\begin{array}{llllll}
1 & 1 & 0 & 1 & 0 & 0
\end{array}\right] .
\end{array}
$$

It may be observed that the process of expressing each row of the matrix $\boldsymbol{X}$ as a join (Boolean sum) of row basis vectors leads to a decomposition of the matrix $\boldsymbol{X}$ of the form:

$$
\boldsymbol{X}=\boldsymbol{U} \boldsymbol{D},
$$

where $\boldsymbol{D}$ is a matrix of dimension $r \times d$ whose rows are the row basis vectors, and where $\boldsymbol{U}$ is an $a \times r$ matrix, with $U_{i j}=1$ if, for each $k=1,2, \ldots, d, X_{i k} \geq D_{j k}$ (that is, if the $i$ th row of $\boldsymbol{X}$ contains the $j$ th row basis vector), and 0, otherwise. Expressing the columns 
of $\boldsymbol{D}$ in terms of its column basis (or, equivalently, the rows of the transpose $\boldsymbol{D}^{\mathrm{T}}$ of $\boldsymbol{D}$ in terms of its row basis) leads to a decomposition of $\boldsymbol{D}$ of the form:

$$
\boldsymbol{D}^{\mathrm{T}}=\boldsymbol{V}^{\mathrm{T}} \boldsymbol{C}^{\mathrm{T}}
$$

where the dimensions of $\boldsymbol{C}$ and $\boldsymbol{V}$ are $r \times c$ and $c \times d$, respectively. We have thus established the following proposition.

Proposition 2. Any a $\times d$ Boolean matrix $\boldsymbol{X}$ possesses a Boolean decomposition of the form:

$$
\boldsymbol{X}=\boldsymbol{U} \boldsymbol{C} \boldsymbol{V}
$$

where $\boldsymbol{U}, \boldsymbol{C}$ and $\boldsymbol{V}$ have dimensions $a \times r, r \times c$, and $c \times d$, respectively, with $r$ being the row rank and $c$ being the column rank of $\boldsymbol{X}$.

Proof. See earlier proof, also Chubb (1986).

The rows and columns of the matrix $\boldsymbol{C}$ are independent; Chubb (1986) termed the matrix $\boldsymbol{C}$ the union-core of the matrix $\boldsymbol{X}$, and described an efficient algorithm for its construction (at polynomial expense). Chubb also established that several important properties of the matrix $\boldsymbol{X}$ are also properties of $\boldsymbol{C}$ : one of these will be described later in detail. It may be observed in passing that the decomposition earlier is somewhat analogous to the Eckart-Young decomposition of a real rectangular matrix (Eckart and Young, 1936), with important differences in the Boolean case being, first, the potential for the row and column ranks to differ and, second, the uniqueness of the row and column bases. We will further discuss these in the following paragraphs.

The decomposition of the matrix $\boldsymbol{X}$ of Table 1 illustrates Proposition 2.

$$
\boldsymbol{X}=\boldsymbol{U} \boldsymbol{D},
$$

where

$$
\boldsymbol{U}=\left[\begin{array}{llll}
0 & 0 & 0 & 0 \\
1 & 0 & 0 & 0 \\
0 & 1 & 0 & 0 \\
0 & 0 & 1 & 0 \\
1 & 0 & 0 & 1 \\
1 & 1 & 0 & 0
\end{array}\right],
$$

and

$$
\boldsymbol{D}=\left[\begin{array}{llllll}
0 & 1 & 0 & 1 & 0 & 0 \\
0 & 0 & 0 & 0 & 1 & 1 \\
1 & 0 & 1 & 1 & 0 & 0 \\
1 & 1 & 0 & 1 & 0 & 0
\end{array}\right]
$$


from which

$$
\boldsymbol{D}=\boldsymbol{C} \boldsymbol{V},
$$

where

$$
\boldsymbol{C}=\left[\begin{array}{llll}
0 & 1 & 0 & 0 \\
0 & 0 & 0 & 1 \\
1 & 0 & 1 & 0 \\
1 & 1 & 0 & 0
\end{array}\right],
$$

and

$$
\boldsymbol{V}=\left[\begin{array}{llllll}
1 & 0 & 0 & 1 & 0 & 0 \\
0 & 1 & 0 & 1 & 0 & 0 \\
1 & 0 & 1 & 1 & 0 & 0 \\
0 & 0 & 0 & 0 & 1 & 1
\end{array}\right]
$$

Proposition 3. The row lattices $L_{\mathrm{R}}(\boldsymbol{X})$ and $L_{\mathrm{R}}(\boldsymbol{D})$ are identical, and both are isomorphic to $L_{\mathrm{R}}(\boldsymbol{C})$. Similarly, $L_{\mathrm{C}}(\boldsymbol{X})$ is isomorphic to $L_{\mathrm{C}}(\boldsymbol{C})$.

Proof. By construction, the matrix $\boldsymbol{D}$ possesses the same row basis as $\boldsymbol{X}$, so that $L_{\mathrm{R}}(\boldsymbol{X})$ and $L_{\mathrm{R}}(\boldsymbol{D})$ are identical. Also, let

$$
f: L_{\mathrm{R}}(\boldsymbol{D}) \rightarrow L_{\mathrm{R}}(\boldsymbol{C}),
$$

be given by

$$
f\left(\vee\left\{\boldsymbol{b}_{a}, a \in S\right\}\right)=\vee\left\{\boldsymbol{c}_{a}, a \in S\right\},
$$

where $\left\{\boldsymbol{b}_{1}, \boldsymbol{b}_{2}, \ldots, \boldsymbol{b}_{r}\right\}$ is the row basis of $\boldsymbol{X}$ (and hence the set of rows of $\left.\boldsymbol{D}\right),\left\{\boldsymbol{c}_{1}, \boldsymbol{c}_{2}, \ldots\right.$, $\left.\boldsymbol{c}_{r}\right\}$ is the row basis of $\boldsymbol{C}, S$ is any subset of the index set $\{1,2, \ldots, r\}$, and $\vee\left\{\boldsymbol{b}_{a}, a \in S\right\}$ denotes the join of $\boldsymbol{b}_{1}, \boldsymbol{b}_{2}, \ldots, \boldsymbol{b}_{r}$. By observing that $\boldsymbol{b}_{i}=\boldsymbol{c}_{i} V$, and that

$$
\left(\vee\left\{\boldsymbol{b}_{a}, a \in S\right\}\right) \vee\left(\vee\left\{\boldsymbol{b}_{e}, e \in T\right\}\right)=\vee\left\{\boldsymbol{b}_{g}, g \in S \cup T\right\},
$$

and

$$
\left(\vee\left\{\boldsymbol{b}_{a}, a \in S\right\}\right) \wedge\left(\vee\left\{\boldsymbol{b}_{e}, e \in T\right\}\right)=\vee\left\{\boldsymbol{b}_{h}, h \in S \cap T\right\},
$$

it may readily be established that $f$ is both a homomorphism and a bijection, so that $L_{\mathrm{R}}(\boldsymbol{C})$ is isomorphic to $L_{\mathrm{R}}(\boldsymbol{D})$.

\subsection{Galois, or concept, lattices}

As Coombs (1964) and others have pointed out, one derives parallel constructions by replacing array entries by their complements, and the addition, or + , operation by the product, or · operation (Doignon et al., 1984). For instance, a row lattice may be defined by 
what Doignon and Falmagne (1985) term a knowledge structure. The row lattice is generated from the rows of $\boldsymbol{X}$ under the union (addition) operation; it is also possible to generate a lattice structure using intersections rather than unions. This strategy is adopted by Wille and colleagues in formal concept analysis (Ganter and Wille, 1989, 1999). The resulting lattice is termed a concept lattice and may be decomposed using the same algorithms as for the union case. Specifically, the following proposition.

Proposition 4. If $M_{\mathrm{R}}(\boldsymbol{X})$ and $M_{\mathrm{C}}(\boldsymbol{X})$ denote the Galois or concept lattice generated by the rows and columns, respectively, of a binary array $X$, then $M_{\mathrm{R}}(X)$ is anti-isomorphic to $L_{\mathrm{R}}\left(\boldsymbol{X}^{\mathrm{c}}\right)$ and $M_{\mathrm{C}}(\boldsymbol{X})$, and isomorphic to $L_{\mathrm{C}}\left(\boldsymbol{X}^{\mathrm{c}}\right)$.

Proof. For example, see Kim (1982) and Boyd (1990).

\subsection{Boolean vector spaces and lattice factorization}

Pattison and Bartlett (1982) introduced a general procedure, termed factorization, for decomposing algebraic structures such as lattices into "maximally independent" components. The procedure is described more formally in Pattison (1993).

Definition. A partition $\theta$ on $L$ is a congruence relation if, for all $x, y, z \in L$, whenever $x \equiv y(\theta)$ then $x \wedge z \equiv y \wedge z(\theta)$ and $x \vee z \equiv y \vee z(\theta)$. Note that we write $x \equiv y(\theta)$ to indicate that $x$ and $y$ are in the same class of the partition $\theta$. Each congruence relation $\theta$ on $L$ corresponds to a lattice homomorphism, that is, to a mapping $\varphi$ on $L$ which satisfies $\varphi(x \wedge z)=\varphi(x) \wedge \varphi(z)$ and $\varphi(x \vee z)=\varphi(x) \vee \varphi(z)$, for all $x, z \in L$.

The congruence relations (and therefore the homomorphisms) of a lattice are themselves partially ordered and form lattices (Pattison, 1993).

Definition. Let $\theta_{1}$ and $\theta_{2}$ be congruence relations on a lattice $L$. If, for all $x, y \in L$, $x \equiv y\left(\theta_{1}\right)$ implies $x \equiv y\left(\theta_{2}\right)$, then we say that $\theta_{1} \leq \theta_{2}$.

The collection of congruence relations of $L$, partially ordered in this way, form a distributive lattice (Birkhoff, 1967, p. 138).

Definition. A lattice $L$ is distributive if the identity

$$
x \wedge(y \vee z)=(x \wedge y) \vee(x \wedge z)
$$

holds. A lattice $L$ is modular if, whenever $x \leq z$, then

$$
x \vee(y \wedge z)=(x \vee y) \wedge z .
$$

Examples of non-distributive and non-modular lattices are displayed in Figs. 2 and 3. The lattice in Fig. 2 is the smallest non-distributive lattice, and it can be shown that every non-distributive lattice contains the lattice of Fig. 2 as a sublattice (that is, as a subset that is also a lattice). The lattices of Figs. 2 and 3 are the smallest non-modular lattices. 


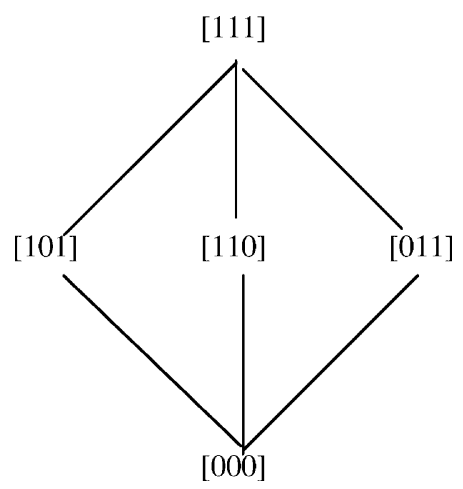

Fig. 2. The lattice $M_{5}$.

It is readily shown (Pattison, 1993) that, for any algebraic structure with a distributive lattice of congruence relations, the decomposition termed factorization (Pattison and Bartlett, 1982) is unique. In particular, for a lattice $L$, factorization is associated with a set of congruence relations on $L$ defined in the following way.

Definition. An atom of a lattice $L$ is any element $a$ that covers the unique minimal element of the lattice (that is, the element $x_{\min }$ satisfying $x \geq x_{\min }$, for all $x \in L$. A meetcomplement of an element $x \in L$ is an element $x^{*}$ such that $x^{*}>x_{\min }$ and $x \wedge x^{*}=x_{\min }$. A meet-complement $x^{*}$ of $x$ is maximal if $x$ has no other meet-complement $z$ such that $z>x^{*}$.

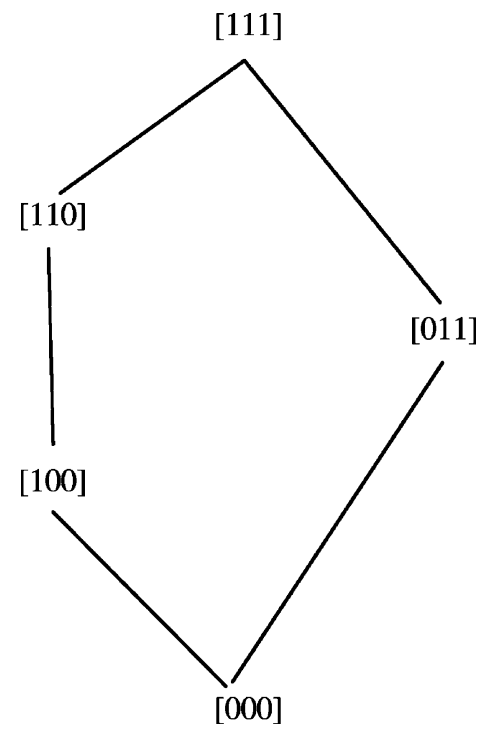

Fig. 3. The lattice $N_{5}$. 
The unique factorization of the lattice $L$ is associated with the set of maximal meetcomplements of the atoms of the congruence lattice of $L$. The homomorphism associated with each congruence relation in this set is a mapping of $L$ onto one of its factors.

We consider below two separate cases, one where the lattice $L_{R}(X)$ is itself distributive, and one where it is not. Since the dual of a distributive lattice is distributive, then either all of $\left\{L_{\mathrm{R}}(\boldsymbol{X}), L_{\mathrm{C}}(\boldsymbol{X}), M_{\mathrm{R}}\left(\boldsymbol{X}^{\mathrm{c}}\right), M_{\mathrm{C}}\left(\boldsymbol{X}^{\mathrm{c}}\right)\right\}$ are distributive or all are not.

\subsection{1. $L_{R}(X)$ is distributive}

In the case where $L_{\mathrm{R}}(\boldsymbol{X})$ itself is distributive, a proposition of Birkhoff (1967) establishes that the components of the factorization are all two-element lattices, each of which may be represented as the lattice denoted by $\mathbf{2}$, and comprising the set $\{0,1\}$ with the ordering $0<1$. Indeed, in this case, $L_{\mathrm{R}}(\boldsymbol{X})$ may be shown to be isomorphic to the lattice $\mathbf{2}^{Z}$, where $Z$ is the partially ordered set of row basis vectors of $L_{\mathrm{R}}(X)$ and where $2^{Z}$ denotes the collection of all isotone mappings from $Z$ onto $\{0,1\}$, partially ordered by the relation $f \leq g$, iff $f(x) \leq g(x)$, for all $x \in Z$.

As a result, each vector $\boldsymbol{x}$ in a distributive row lattice $L_{R}(\boldsymbol{X})$ may be represented by a binary vector of dimension $r$ corresponding to the factorization: the $i$ th component of the vector corresponding to $\boldsymbol{x}$ is equal to 1 if and only if $\boldsymbol{x} \geq \boldsymbol{b}_{i}$. Thus, in the case where $L_{\mathrm{R}}(\boldsymbol{X})$ is distributive, factorization of $L_{\mathrm{R}}(\boldsymbol{X})$ yields a decomposition of $\boldsymbol{X}$ identical to that of Proposition 2, and hence a Boolean decomposition of the form $\boldsymbol{X}=\boldsymbol{U} \boldsymbol{C} \boldsymbol{V}$.

Proposition 5. Let $L_{\mathrm{R}}(\boldsymbol{X})$ be a distributive lattice with row basis $\left\{\boldsymbol{b}_{1}, \boldsymbol{b}_{2}, \ldots, \boldsymbol{b}_{r}\right\}$. Then, the factorization of $L_{\mathrm{R}}(\boldsymbol{X})$ expresses each lattice element $\boldsymbol{x}$ as a binary vector $\left[\begin{array}{lllll}x_{1} & x_{2} & \cdots & x_{\mathrm{r}}\end{array}\right]$, where $x_{i}=1$ if $\boldsymbol{x} \geq \boldsymbol{b}_{i} ; 0$, otherwise. As a result, $\boldsymbol{X}$ possesses a decomposition induced by the factorization of the form

$$
\boldsymbol{X}=\boldsymbol{U} \boldsymbol{C}
$$

Proof. The lattice $L_{\mathrm{R}}(\boldsymbol{X})$ has $r$ join-irreducible elements forming a partially ordered set $Z$ and, since $L_{\mathrm{R}}(\boldsymbol{X})$ is distributive, $L_{\mathrm{R}}(\boldsymbol{X})$ is isomorphic to $2^{Z}$. Consequently, the factors of $L_{\mathrm{R}}(\boldsymbol{X})$ correspond to congruence relations $\sigma_{i}$ of the form

$$
\left(\boldsymbol{x}, \boldsymbol{b}_{i}\right) \in \sigma_{i}, \quad \text { iff } \boldsymbol{x} \geq \boldsymbol{b}_{i},
$$

and

$$
(\boldsymbol{x}, \boldsymbol{y}) \in \sigma_{i}, \quad \text { if } \boldsymbol{x} \wedge \boldsymbol{b}_{i}=\boldsymbol{y} \wedge \boldsymbol{b}_{i}=0,
$$

so that the $i$ th co-ordinate of $\boldsymbol{x}$ in the factorization of $L_{\mathrm{R}}(\boldsymbol{X})$ is equal to 1 if $\boldsymbol{x} \geq \boldsymbol{b}_{i}$, and 0 , otherwise. Thus, $\boldsymbol{X}=\boldsymbol{U} \boldsymbol{D}$, where $\boldsymbol{D}$ is a matrix whose rows are the basis vectors $\boldsymbol{b}_{1}, \boldsymbol{b}_{2}$, $\ldots, \boldsymbol{b}_{r}$. Further, since $L_{\mathrm{R}}(\boldsymbol{X})$ is distributive, it follows from Proposition 4 that $L_{\mathrm{R}}(\boldsymbol{D})$ and hence also $L_{C}(\boldsymbol{D})$ are distributive, and the result may be applied to the transpose of $\boldsymbol{D}$ to obtain the required decomposition.

For example, the binary matrix $\boldsymbol{Y}$ presented in Table 3 possesses the row lattice $M$ shown in Fig. 4, and it may readily be verified that the lattice is distributive. The factorization of 
Table 3

The binary matrix $\boldsymbol{Y}$

\begin{tabular}{llll}
\hline 1 & 1 & 0 & 0 \\
1 & 1 & 0 & 1 \\
0 & 0 & 0 & 0 \\
0 & 1 & 1 & 1 \\
1 & 1 & 1 & 1 \\
0 & 0 & 0 & 1 \\
0 & 1 & 1 & 0 \\
1 & 1 & 1 & 0 \\
\hline
\end{tabular}

$M$ is associated with the following congruence relations:

$$
\begin{aligned}
& \sigma_{1}:\left(\begin{array}{llll}
3 & 4 & 6 & 7
\end{array}\right)\left(\begin{array}{llll}
1 & 2 & 5 & 8
\end{array}\right), \\
& \sigma_{2}:\left(\begin{array}{llll}
1 & 2 & 3 & 6
\end{array}\right)\left(\begin{array}{llll}
4 & 5 & 7 & 8
\end{array}\right), \\
& \sigma_{3}:\left(\begin{array}{llll}
1 & 3 & 7 & 8
\end{array}\right)\left(\begin{array}{llll}
2 & 4 & 5 & 6
\end{array}\right),
\end{aligned}
$$

so that, each lattice element may be represented as a binary vector of dimension three as shown in Table 4. The associated decomposition of $\boldsymbol{Y}$ is therefore:

$\boldsymbol{Y}=\boldsymbol{U} \boldsymbol{D}$

(5) $[1111]$
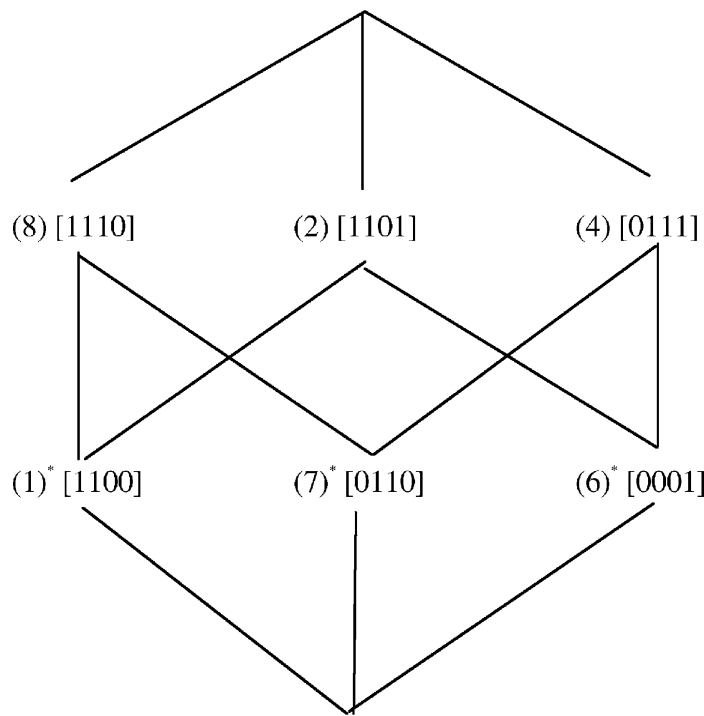

(3) [0000]

Fig. 4. The row lattice $L_{\mathrm{R}}(\boldsymbol{Y})$ (join-irreducible elements are marked by an asterisk). 
Table 4

Representation of elements of the lattice $M$ induced by factorization of $L_{\mathrm{R}}(M)$

\begin{tabular}{|c|c|c|}
\hline Lattice element & Vector in $L_{\mathrm{R}}(M)$ & Induced representation \\
\hline 1 & {$\left[\begin{array}{llll}1 & 1 & 0 & 0\end{array}\right]$} & $(1,0,0)$ \\
\hline 2 & {$\left[\begin{array}{llll}1 & 1 & 0 & 1\end{array}\right]$} & $(1,0,1)$ \\
\hline 3 & {$\left[\begin{array}{llll}0 & 0 & 0 & 0\end{array}\right]$} & $(0,0,0)$ \\
\hline 4 & {$\left[\begin{array}{llll}0 & 1 & 1 & 1\end{array}\right]$} & $(0,1,1)$ \\
\hline 5 & {$\left[\begin{array}{llll}1 & 1 & 1 & 1\end{array}\right]$} & $(1,1,1)$ \\
\hline 6 & {$\left[\begin{array}{llll}0 & 0 & 0\end{array}\right]$} & $(0,0,1)$ \\
\hline 7 & {$\left[\begin{array}{llll}0 & 1 & 1 & 0\end{array}\right]$} & $(0,1,0)$ \\
\hline 8 & {$\left[\begin{array}{llll}1 & 1 & 1 & 0\end{array}\right]$} & $(1,1,0)$ \\
\hline
\end{tabular}

where

$$
\boldsymbol{U}=\left[\begin{array}{lll}
1 & 0 & 0 \\
1 & 1 & 0 \\
0 & 0 & 0 \\
0 & 1 & 1 \\
1 & 1 & 1 \\
0 & 1 & 0 \\
0 & 0 & 1 \\
1 & 0 & 1
\end{array}\right]
$$

and

$$
\boldsymbol{D}=\left[\begin{array}{llll}
1 & 1 & 0 & 0 \\
0 & 0 & 0 & 1 \\
0 & 1 & 1 & 0
\end{array}\right],
$$

so that

$$
\boldsymbol{C}=\left[\begin{array}{lll}
1 & 0 & 0 \\
0 & 0 & 1 \\
0 & 1 & 0
\end{array}\right],
$$

and

$$
\boldsymbol{V}=\left[\begin{array}{llll}
1 & 1 & 0 & 0 \\
0 & 1 & 1 & 0 \\
0 & 0 & 0 & 1
\end{array}\right]
$$

It may be observed that the binary matrix component corresponding to the $i$ th factor in the factorization divides the rows of $\boldsymbol{X}$ into two groups: those rows which lie above the corresponding row basis vector and those that do not. The columns determining this division are those having unit entries in the associated basis vector. This observation leads to a more general construction, described in Section 2.4.

\subsection{2. $L_{R}(\boldsymbol{X})$ is not distributive}

In the case where $L_{R}(X)$ is not distributive, at least one of the factors of $L_{R}(X)$ will not be isomorphic to the two-element lattice $\mathbf{2}$. For example, the lattice presented in Table 2 
(and Fig. 1) has factors isomorphic to 2 and $N_{5}$; these factors correspond to the congruence relations ( $\left.1 \begin{array}{lllll}1 & 2 & 4 & 5 & 7\end{array}\right)\left(\begin{array}{lllll}3 & 6 & 8 & 9 & 10\end{array}\right)$ and $\left(\begin{array}{ll}1 & 3\end{array}\right)\left(\begin{array}{ll}2 & 6\end{array}\right)\left(\begin{array}{ll}4 & 8\end{array}\right)\left(\begin{array}{ll}5 & 9\end{array}\right)$ ( 710 ) of $L_{R}(\boldsymbol{X})$, respectively. Later, we describe a general decomposition of an array that is associated with any homomorphism of its row lattice, and hence with the factors in its factorization.

\subsection{Induced decompositions of a data array}

It is natural to ask whether, if the lattice $L_{\mathrm{R}}(\boldsymbol{X})$ has a homomorphic image $K$ (such as one of the factors of $L$ ), can we find one or more "reductions" of the data array that is consistent with the congruence relation for the homomorphism and that generates the lattice $K$ ? If so, we can argue that there is an association between such a reduction of the array and the homomorphic image.

Proposition 6. Suppose that $H$ is a homomorphic image of $L_{\mathrm{R}}(\boldsymbol{X})$ with associated congruence relation $\psi$ and that $K$ is a homomorphic image of $M_{\mathrm{R}}(\boldsymbol{X})$ with associated congruence relation $\theta$. For each row vector $\boldsymbol{x}$ in the matrix $\boldsymbol{X}$, define

$$
\boldsymbol{x}_{\psi}=\wedge\left\{\boldsymbol{y}: \boldsymbol{x} \equiv \boldsymbol{y}(\psi), \quad \text { for } \boldsymbol{x}, \boldsymbol{y} \in L_{\mathrm{R}}(\boldsymbol{X})\right\},
$$

and

$$
\boldsymbol{x}^{\theta}=\vee\left\{\boldsymbol{y}: \boldsymbol{x} \equiv \boldsymbol{y}(\theta), \quad \text { for } \boldsymbol{x}, \boldsymbol{y} \in M_{\mathrm{R}}(\boldsymbol{X})\right\} .
$$

Also define $\boldsymbol{X}_{\psi}$ and $\boldsymbol{X}^{\theta}$ to be the matrices in which the row $\boldsymbol{x}$ of the array $\boldsymbol{X}$ is replaced by $\boldsymbol{x}_{\psi}$ and $\boldsymbol{x}^{\theta}$, respectively. Then $L_{\mathrm{R}}\left(\boldsymbol{X}_{\psi}\right)$ is isomorphic to $H$ and $M_{\mathrm{R}}\left(\boldsymbol{X}^{\theta}\right)$ is isomorphic to $K$.

Proof (See also Ganter and Wille (1999)). There is clearly a one-to-one relationship between elements of $H$ and elements of $L_{\mathrm{R}}(\boldsymbol{X})$, and likewise for $K$ and $M_{\mathrm{R}}(\boldsymbol{X})$; in addition each construction leads to a homomorphism, since $(\boldsymbol{x} \wedge \boldsymbol{z})_{\psi}=\boldsymbol{x}_{\psi} \wedge \boldsymbol{z}_{\psi}$ and $(\boldsymbol{x} \vee \boldsymbol{z})^{\theta}$ $=\boldsymbol{x}^{\theta} \vee \boldsymbol{z}^{\theta}$.

Note that, by construction, both $\boldsymbol{X}_{\psi}$ and $\boldsymbol{X}^{\theta}$ have a reducible form, so that Proposition 2 and its intersection analogue can be applied to identify the union-core $\boldsymbol{C}_{\psi}$ of $\boldsymbol{X}_{\psi}$ and the intersection-core $\boldsymbol{C}^{\theta}$ of $\boldsymbol{X}^{\theta}$ which also have lattices that are isomorphic to $H$ and $K$, respectively.

\subsection{Boolean factor analysis}

Where $L_{\mathrm{R}}(\boldsymbol{X})$ is not a distributive matrix, factorization does not yield a Boolean matrix product representation of $\boldsymbol{X}$. It has been observed, however, in the literature that such representations are of interest (De Boeck and Rosenberg, 1988; Mickey et al., 1983) and now we consider them in relation to $L_{R}(X)$ and its factorization.

One general form of these representations that has been studied is a Boolean analogue of factor analysis, that is, an expression of $\boldsymbol{X}$ as a Boolean product of matrices $\boldsymbol{P}$ and $\boldsymbol{Q}$ :

$$
X=P Q .
$$


Definition. The Schein rank $s=s(\boldsymbol{X})$ of an $a \times d$ binary matrix $\boldsymbol{X}$ is the least integer $s$ such that $\boldsymbol{X}$ may be expressed as a Boolean product of matrices $\boldsymbol{P}$ and $\boldsymbol{Q}$ of dimension $a \times s$ and $s \times d$, respectively.

Some well-known equivalent definitions of the Schein rank of a matrix are summarized in Proposition 7.

Proposition 7 (Kim (1982)). The following are equivalent:

(a) the Schein rank of $\boldsymbol{X}$ is $s$;

(b) $s$ is the least integer such that $\boldsymbol{X}$ may be expressed as the Boolean sum of s cross-vectors (or rectangles), that is of matrices of the form $\boldsymbol{C}=\left[\begin{array}{llll}v_{1} & v_{2} & \cdots & v_{a}\end{array}\right]^{\mathrm{T}}\left[\begin{array}{llll}w_{1} & w_{2} & \cdots\end{array}\right.$ $\left.w_{d}\right]$

(c) $s$ is the least integer such that $L_{\mathrm{R}}(X)$ is a subset of a subspace $W$ of $V_{d}$ spanned by $s$ vectors;

(d) $s$ is the least integer such that $L_{\mathrm{C}}(\boldsymbol{X})$ is a subset of a subspace $W$ of $V_{a}$ spanned by $s$ vectors.

For instance, a Boolean factor analysis

$$
\boldsymbol{X}=\boldsymbol{P} \boldsymbol{Q}
$$

may be converted into a cross-vector representation by putting

$$
\boldsymbol{P}=\left[\begin{array}{llll}
\boldsymbol{p}_{1} & \boldsymbol{p}_{2} & \cdots & \boldsymbol{p}_{s}
\end{array}\right],
$$

where $\boldsymbol{p}_{i}$ is a column vector of dimension $a$, and

$$
\boldsymbol{Q}=\left[\begin{array}{llll}
\boldsymbol{q}_{1} & \boldsymbol{q}_{2} & \cdots & \boldsymbol{q}_{s}
\end{array}\right],
$$

where $\boldsymbol{q}_{i}$ is a row vector of dimension $d$, so that

$$
\boldsymbol{P} \boldsymbol{Q}=\boldsymbol{p}_{1} \boldsymbol{q}_{1}+\boldsymbol{p}_{2} \boldsymbol{q}_{2}+\cdots+\boldsymbol{p}_{s} \boldsymbol{q}_{s},
$$

and each $\boldsymbol{p}_{i} \boldsymbol{q}_{i}$ is a cross vector.

Doignon and Falmagne (1984) described these representations in terms of matching relations. A binary matrix $\boldsymbol{X}$ is a matching relation if it is a cross vector, that is, if $X_{i k}=$ $1=X_{j k}=X_{j h}$ implies $X_{i h}=1$. For binary proximity relations, a matching relation may be regarded as a type of two-set cluster, linking a subset of row entities to a subset of column entities. Thus, the representation described by Doignon and Falmagne expresses a proximity relation as the union of possibly overlapping clusters of this type.

It is useful once more to discuss separately those cases where $L_{\mathrm{R}}(\boldsymbol{X})$ is distributive and those where it is not.

\subsection{1. $L_{R}(\boldsymbol{X})$ distributive}

Proposition 8. If $L_{\mathrm{R}}(\boldsymbol{X})$ is distributive, then $s(\boldsymbol{X})=r(\boldsymbol{X})=c(\boldsymbol{X})$ and the decomposition

$$
\boldsymbol{X}=\boldsymbol{U} \boldsymbol{D},
$$

yields a Boolean factor analysis of $\boldsymbol{X}$ into matrices of dimension $a \times s$ and $s \times d$. 
Proof. Since $L_{\mathrm{R}}(\boldsymbol{X})$ is isomorphic to $\mathbf{2}^{Y}$ where $Y$ is the partially ordered set of basis vectors of $\boldsymbol{X}, L_{\mathrm{R}}(\boldsymbol{X})$ has a chain (that is a sequence of distinct elements $x_{0}<x_{1}<\ldots<x_{r}$ ) comprising $r+1$ elements (Birkhoff, 1967). As a result, at least $r$ vectors are required in any subset of $V_{d}$ whose span contains $L_{\mathrm{R}}(\boldsymbol{X})$. Thus, $s \geq r$. For any matrix $\boldsymbol{X}, r \geq s$ (Kim, 1982), and so we have established that $r=s$ and that the decomposition $\boldsymbol{X}=\boldsymbol{U} \boldsymbol{D}$ is a Boolean factor analysis. The result that $c=s$ follows by replacing $\boldsymbol{X}$ by its transpose in the argument earlier.

Thus, in the case of a distributive row lattice, the Schein rank of $\boldsymbol{X}$ is equal to its row rank, and the constructions we have already discussed yield a corresponding Boolean factor analysis.

\subsection{2. $L_{R}(X)$ not distributive}

The case of non-distributivity is more interesting, since $L_{R}(X)$ can be embedded as a partial order in a subspace of $V_{d}$ having a smaller basis than $L_{\mathrm{R}}(\boldsymbol{X})$ itself. Later, we give an embedding rule which guarantees that the basis of the space into which $L_{R}(\boldsymbol{X})$ is embedded has a minimal number of vectors. We then show that this space must be distributive, so that each row of $\boldsymbol{X}$ can be expressed via factorization as an element in $\mathbf{2}^{s}$. The expression therefore yields a Boolean decomposition of $\boldsymbol{X}$ into matrices of dimension $a \times s$, and $s \times d$.

Proposition 9. Let $\boldsymbol{B}$ be the row basis of a matrix $\boldsymbol{X}$, partially ordered by

$$
\boldsymbol{b}_{i} \leq \boldsymbol{b}_{j}, \quad \text { iff } b_{i k} \leq b_{j k}, \quad \text { for all } k \in\{1,2, \ldots, d\} .
$$

Let $\boldsymbol{b}_{j} \in B$ be some vector for which $\boldsymbol{b}_{i} \leq \boldsymbol{b}_{j}$ and $\boldsymbol{b}_{i} \neq \boldsymbol{b}_{j}$ for some $\boldsymbol{b}_{i}$. Then, the space spanned by $B$ is contained in the space spanned by

$$
B^{\prime}=B \backslash\left\{\boldsymbol{b}_{j}\right\} \text { union }\left\{\boldsymbol{x}_{j}\right\},
$$

where $\boldsymbol{x}_{j}$ is a vector such that $\boldsymbol{x}_{j} \vee \boldsymbol{B}_{j}=\boldsymbol{b}_{j}$ and $\boldsymbol{x}_{j} \wedge \boldsymbol{B}_{j}=\mathbf{0}$, and $\boldsymbol{B}_{j}$ is the vector

$$
B_{j}=\vee\left\{\boldsymbol{b}_{i}: \boldsymbol{b}_{i} \leq \boldsymbol{b}_{j} \text { and } \boldsymbol{b}_{i} \neq \boldsymbol{b}_{j}\right\}
$$

Proof. By construction, $\boldsymbol{b}_{j}=\boldsymbol{B}_{j} \vee \boldsymbol{x}_{j}$, hence the span of $B$ is contained in the span of $B^{\prime}$.

This construction leads to the following algorithm.

Algorithm. Set $W=B$, and arrange the basis vectors in $W$ in some order. Consider elements of $W$ in turn. If $\boldsymbol{w}_{j}$ is minimal in $W$, leave it unchanged; if $\boldsymbol{w}_{j}$ is not minimal, construct the corresponding vector $\boldsymbol{x}_{j}$ of the preceding proposition. If $\boldsymbol{x}_{j}$ is equal to any of the existing $\boldsymbol{w}_{i}\left(\boldsymbol{w}_{i} \neq \boldsymbol{w}_{j}\right)$, discard it from $W$. Repeat the process through cycles of elements of $W$, until an entire cycle through $W$ leaves all of its members unchanged.

Proposition 10. The algorithm constructs an independent set $W$ containing s vectors, where $s=s(\boldsymbol{X})$ is the Schein rank of $\boldsymbol{X}$. 
Proof. Any set spanning a set $W$, but smaller than $W$ must have elements lying between members of $W$ and the zero vector in $V_{d}$. By construction, however, each element of $W$ is minimal, and so the cardinality of $W$ is minimal.

Proposition 11. The space spanned by the set $W$ resulting from the algorithm above is distributive.

Proof. Since elements of $W$ possess no partial ordering, the space spanned by $W$ is isomorphic to $\mathbf{2}^{W}$ and is therefore distributive.

Thus, the construction amounts to an embedding of $L_{\mathrm{R}}(\boldsymbol{X})$ as a partial order in a distributive space, and the latter possesses a factorization into $s$ components all of which are isomorphic to the lattice 2 . Each row of the matrix $\boldsymbol{X}$ may therefore be expressed as a binary vector of dimension $s$ and the algorithm therefore constructs a decomposition of $\boldsymbol{X}$ of the form

$$
\boldsymbol{X}=\boldsymbol{P} \boldsymbol{Q},
$$

The $s$ rows of $\boldsymbol{Q}$ are the $s$ vectors in $W$, and $P_{i j}=1$ if $X_{i k} \geq W_{j k}$ for all $k \in\{1,2, \ldots, d\}$ and 0 , otherwise. Of course, it will often be more efficient to apply this procedure directly to the union-core matrix $\boldsymbol{C}$ of the decomposition of Proposition 2, yielding,

$$
\boldsymbol{C}=\boldsymbol{P}^{\prime} \boldsymbol{Q}^{\prime}
$$

and so leading to the representation of $\boldsymbol{X}$ in the form:

$$
\boldsymbol{X}=\boldsymbol{U} \boldsymbol{P}^{\prime} \boldsymbol{Q}^{\prime} \boldsymbol{V}
$$

and hence

$$
\boldsymbol{X}=\boldsymbol{P} \boldsymbol{Q},
$$

where $\boldsymbol{P}=\boldsymbol{U} \boldsymbol{P}^{\prime}$ and $\boldsymbol{Q}=\boldsymbol{Q}^{\prime} \boldsymbol{V}$. The result that the Schein rank of $\boldsymbol{X}$ is the same as the Schein rank of $\boldsymbol{C}$ was also established by Chubb (1986).

The constructions earlier make it clear that the distinction between observed and latent variable representations may be applied to the two different types of decomposition of a matrix that we have been considering. In the decomposition of a matrix $\boldsymbol{X}$ in the form

$$
\boldsymbol{X}=\boldsymbol{U} \boldsymbol{D},
$$

each row of $\boldsymbol{X}$ is expressed in terms of "observed" row basis vectors. By contrast, the decomposition of $\boldsymbol{X}$ in the form

$$
\boldsymbol{X}=\boldsymbol{P} \boldsymbol{Q},
$$

expresses each row of $\boldsymbol{X}$ in terms of the set $W$ of "latent" vectors which are not necessarily observed as rows of $\boldsymbol{X}$ but which have the capacity to generate all of the observed rows of $\boldsymbol{X}$.

One final question that we consider here is the relationship between alternative Boolean factor analyses of a matrix $\boldsymbol{X}$. We demonstrate that the earlier construction leads 
to a maximal Boolean factor analysis of the matrix $\boldsymbol{X}$, an analysis that has been termed a set-theoretical decomposition by De Boeck and Rosenberg (1988). We first give the following definition.

Definition. Let $\boldsymbol{X}=\boldsymbol{P} \boldsymbol{Q}$ and $\boldsymbol{X}=\boldsymbol{P}^{\prime} \boldsymbol{Q}^{\prime}$ be alternative Boolean factor analyses of $\boldsymbol{X}$ (so that both $\boldsymbol{P}$ and $\boldsymbol{P}^{\prime}$ have dimension $a \times s$, and $\boldsymbol{Q}$ and $\boldsymbol{Q}^{\prime}$ have dimension $s \times d$ ). Let $[\boldsymbol{P}, \boldsymbol{Q}] \leq\left[\boldsymbol{P}^{\prime}, \boldsymbol{Q}^{\prime}\right]$ if $P_{i j} \leq P_{i j}^{\prime}$, for all $i, j$ and $Q_{i j} \leq Q_{i j}^{\prime}$, for all $i, j$.

Proposition 12. Let $\boldsymbol{X}=\boldsymbol{P} \boldsymbol{Q}$ be a Boolean factor analysis of $\boldsymbol{X}$. Then, for each matrix $\boldsymbol{P}$ in such a decomposition, there is a maximal matrix $\boldsymbol{Q}^{*}$ satisfying $\boldsymbol{X}=\boldsymbol{P} \boldsymbol{Q}^{*}$ and for each matrix $\boldsymbol{Q}$ there is a maximal $\boldsymbol{P}^{*}$ satisfying $\boldsymbol{X}=\boldsymbol{P}^{*} \boldsymbol{Q}$.

Further, the procedure based on Proposition 10 yields this unique maximal solution.

Proof. The result follows directly from the existence of unique maximal solutions to Boolean relational equations (Sanchez, 1976).

\section{Non-binary representations}

Suppose now that the values taken by $X_{i j}$ are not binary but from a set $A$ of values. We assume that $A$ includes a minimum value of 0 and a maximum value of 1 .

\subsection{Matrices over a general Boolean algebra}

Define the operations,$+ \cdot$ and ${ }^{\mathrm{c}}$ by: (i) $a+b=\max (a, b)$; (ii) $a . b=\min (a, b)$; and (iii) $a^{\mathrm{c}}=1-a$. Then $A$ is a Boolean algebra and most of the results described earlier can be directly generalized to this case (see Appendix in Kim (1982)). In particular, row and column bases of the resulting Boolean vector spaces are unique, and the constructions just described lead to lattices whose join-irreducible elements correspond to basis elements.

\subsection{Matrices over a fuzzy algebra}

If, on the other hand, we assume that $A=[0,1]$ and that the operations + and '. are the maximum and minimum operations just defined, then $A$ is a fuzzy algebra. In this case, $A$ is not necessarily finite, and the important difference between this case and the case of matrices over an arbitrary Boolean algebra is that bases of the vector spaces to which they give rise are not unique (Kim, 1982).

\subsection{Incline algebras}

More generally, $A$ is an incline algebra if it has (i) an associative, commutative addition operation ' + ', (ii) a distributive product operation '.', and (iii) if $a+a=a$ and $a+a b=a$ for all $a, b \in A$. Matrices over an incline form a semiring; we can define row and column 
bases, as well as standard bases, and there are some results on when the bases (or standard bases) are unique (Cao et al., 1984).

\subsection{Matrices over the reals}

The final case is a familiar one and the theory of real vector spaces, bases, and standard bases are well-known. A fundamental mathematical result in this case is the Eckart-Young decomposition of a real-valued $n \times m$ array $\boldsymbol{X}$ (Young and Householder, 1938) in the form $\boldsymbol{X}=\boldsymbol{X}^{*} \boldsymbol{\Lambda} \boldsymbol{V}^{*^{\prime}}$, where $\boldsymbol{U}^{*}$ and $\boldsymbol{V}^{*}$ are $n \times p$ and $m \times p$ orthogonal matrices, $\boldsymbol{\Lambda}$ is a diagonal matrix, with entries $\left(\lambda_{1}, \lambda_{2}, \ldots, \lambda_{p}\right)$, and $p$ is the eigendimension of $\boldsymbol{X}$. Expressing $\boldsymbol{U}^{*}$ and $\boldsymbol{V}^{*}$ in terms of orthonormal matrices $\boldsymbol{U}$ and $\boldsymbol{V}$ via $\boldsymbol{U}^{*}=\boldsymbol{D}_{1}^{-1} \boldsymbol{U}$ and $\boldsymbol{V}^{*}=\boldsymbol{D}_{2}^{-1} \boldsymbol{V}$ leads to the form $\boldsymbol{X}=\boldsymbol{D}_{1}^{-1} \boldsymbol{U} \boldsymbol{\Lambda} \boldsymbol{V}^{\prime} \boldsymbol{D}_{2}^{-1}$, where the rows of $\boldsymbol{U}$ and $\boldsymbol{V}$ may be regarded as an orthonormal basis of the vector space spanned by the rows and columns of $\boldsymbol{X}$, respectively. Indeed, the proof of the Eckart-Young decomposition establishes a one-to-one correspondence between the matrix decomposition and the expression of the rows and columns of $\boldsymbol{X}$ in terms of orthonormal row and column bases. Since each element $X_{i j}$ of $\boldsymbol{X}$ is thereby represented in the form $X_{i j}=\left(D_{1}^{-1}\right)_{i i}\left(D_{2}^{-1}\right)_{j j} \sum_{k} \lambda_{k}\left(U_{i k} V_{j k}\right)$, it has been common to represent the decomposition in terms of a mapping of the entities indexed by both the rows and the columns of $\boldsymbol{X}$ in a $p$-dimensional real space.

\section{Reduced rank representations}

The discussion so far has focused on the problem of obtaining "exact" decompositions for an array $\boldsymbol{X}$. An implicit assumption of the constructions that have been analyzed is that the data are measured without error and hence that it is desirable to represent every feature possessed by the data in the decomposition. Needless to say, this is often an unreasonable assumption and there is much to recommend attempts to obtain approximate representations using "small" numbers of "simple" components. Indeed, the reasons for pursuing an understanding of algebraic decomposition techniques include (a) the development of exact representational strategies for comparison with existing or new proposed approximate techniques, and (b) the hope of obtaining some insights for the development of new approximate methods (Schönemann, 1970).

There are at least two different approaches to the development of new approximate techniques. One is a more formal, "confirmatory", statistical approach and involves specifying a probability model for an array of binary random variables. The Rasch model and a number of latent class models (Feick, 1987; Rindskopf, 1983; Martin and Wiley, 1999, 2000) relating binary response profiles to one or a small number of biorder components are good examples of the approach; random bipartite graph models (Skvoretz and Faust, 1999; Pattison and Robins, 2000) are another. In this approach, the general form of the model is assumed to be known and the data are used to estimate model parameters.

An alternative strategy is to adopt a more exploratory stance, seeking fewer components than the number required for exact representation, but often more than is allowed by a probabilistic approach to the problem. Algorithms for reduced rank decompositions in real vector 
spaces are of course well-known. Decompositions can be based on correspondence analysis, loglinear and association models, latent class analysis, multidimensional scaling and related techniques (Blasius and Greenacre, 1998). Several exploratory methods for approximate Boolean factor analysis also exist (Mickey et al., 1983; De Boeck and Rosenberg, 1988). Here, we briefly review these Boolean approximate techniques and we also describe some alternative strategies for fitting approximate decompositions of the form that we have been considering.

\subsection{Algorithms for approximate Boolean decompositions of the form $\boldsymbol{X}=\boldsymbol{U} \boldsymbol{C} \boldsymbol{V}$}

One strategy for fitting approximate decompositions is to perform an exact analysis (as described earlier) and then to attach to each component of the resulting decomposition some measure of the "extent" of the data covered by the component. One might then proceed in a stepwise manner (for instance, backward or forward) to identify a smaller number of components which cover "most" of the data. Presumably, a large number of "extent" measures could be proposed, but one desirable feature that any selected measure should possess is some consistency with the metric implicit in the lattice $L_{\mathrm{r}}(\boldsymbol{X})$ associated with the algebra representing the data matrix.

A second general strategy is to simplify the algebra representing the data prior to decomposition. No such strategy has been suggested for row or column lattices, but eliminating row basis vectors required by only a small fraction of the observed rows may be a useful starting point. Indeed, for rectangular data, using the outcome of either of these two strategies to obtain a representation with a small number of components would provide a useful starting configuration for the Boolean regression step of Boolean factor analysis (Mickey et al., 1983; De Boeck and Rosenberg, 1988).

In some cases, it might also be possible to identify a single "best" component for a data array, a process that could be iteratively re-applied to a residual array constructed from the data and the components fitted so far. Such a strategy, for instance, is incorporated in the qualitative factor analysis methods described by Mirkin (1987) for non-negative symmetric proximity matrices.

\subsection{Algorithms for Boolean factor analysis}

Mickey et al. (1983) described an algorithm for Boolean factor analysis that is based on Boolean regression procedures. The algorithm assumes that the observed binary array $\boldsymbol{X}$ may be expressed in the form

$$
\boldsymbol{X}=\boldsymbol{P} \boldsymbol{Q}+\boldsymbol{E},
$$

where $\boldsymbol{P}$ and $\boldsymbol{Q}$ are unknown binary matrices of dimension $a \times s$ and $s \times d$, respectively, and the matrix $\boldsymbol{E}$ has entries of $-1,0$ or 1 . The sum of absolute values of entries in $\boldsymbol{E}$ can be regarded as a measure of discrepancy between the data matrix $\boldsymbol{X}$ and the Boolean factor analysis solution $\boldsymbol{P} \boldsymbol{Q}$. Mickey et al.'s algorithm begins with an initial estimate for the matrix $\boldsymbol{Q}$ and then estimates the rows of $\boldsymbol{P}$ using an algorithm for Boolean regression. Once the rows of $\boldsymbol{P}$ have been estimated, the columns of $\boldsymbol{Q}$ are estimated using the same 
technique, and the whole process is recycled several times, with several different values of $s$ in a pre-specified range and in a pre-arranged order (Mickey et al., 1983). De Boeck and Rosenberg (1988) adapted the Boolean factor analysis algorithm to fit approximate 'set-theoretical decompositions' to an array $\boldsymbol{X}$ : the result is an algorithm termed HICLAS.

It may be noted that the development of analytic methods for discrete structural models lags well behind that for the more traditional linear models used in the behavioral and social sciences. One reason for this delay is the greater complexity of structural models, and their foundation in more general (and, as yet, less tractable) algebraic structures than the linear algebras underlying the traditional linear models. Yet, procedures for expressing a binary array in terms of discrete, binary matrix components have two noteworthy properties as methods of data analysis. First, they seek components of the original data which have the same form as those original data, a property that they share with techniques like blockmodelling (Arabie et al., 1978; Breiger et al., 1975; White et al., 1976). Second, the components are derived using a small set of simple Boolean operations, such as union, intersection, relational composition, and binary comparison. Restricting the data analyses to such operations means that only a minimal set of assumptions need be made about the measurement characteristics of the data, and, at the very least, the procedures that result will provide a useful comparative base for those techniques which make a different and often stronger set of assumptions about the data.

\section{Future directions}

This paper has been concerned with some methods that attempt to provide simultaneous representation of dual relationships. We have demonstrated fundamental similarities among these approaches. These similarities provide foundations for research examples that seek to combine dimensional representations and lattices within the same substantive study (Wolff and Gabler, 1998; Breiger, 2000, pp. 102-106).

Algorithms for reduced rank fuzzy decompositions remain an open problem. An additional topic of interest is the relation of probabilistic models to those we have discussed. Skvoretz and Faust (1999) formulate models allowing study of the (log) odds of an actor's belonging to an event (or an event including an actor) as a function of properties of the two-mode network of actors' memberships in events, based on the $\boldsymbol{P}^{*}$ family of models. Pattison and Robins (2000) formulate neighborhood-based models for dual networks that extends this line of research by allowing a contingency to be created between the two possible membership ties by virtue of their connection to an observed tie, hence allowing for a collection of attendance ties to become mutually contingent (Pattison and Robins, in press). Future work should build on these approaches as well as on Breiger (2000) to use lattice techniques to simplify the structure of probabilities of relations in dual networks.

\section{References}

Arabie, P., Boorman, S.A., Levitt, P.R., 1978. Constructing blockmodels: how and why. Journal of Mathematical Psychology 17, 21-63.

Arabie, P., Carroll, J.D., DeSarbo, W., 1987. Three-way Scaling and Clustering. Sage, Beverly Hills, CA. 
Birkhoff, G., 1967. Lattice Theory, 3rd Edition. American Mathematical Society, Providence, RI.

Blasius, J., Greenacre, M., 1998. Visualization of Categorical Data. Academic Press, San Diego.

Boyd, J.P., 1990. Social Semigroups: A Unified Theory of Scaling and Blockmodelling as Applied to Social Networks. George Mason University Press, Fairfax, VA.

Breiger, R.L., 1974. The duality of persons and groups. Social Forces 53, 181-190.

Breiger, R.L., 1990. Social control and social networks: a model from Georg Simmel. In: Calhoun, C., Meyer, M.W., Scott, W.R. (Eds.), Structures of Power and Constraint: Papers in Honor of Peter M. Blau. Cambridge University Press, Cambridge, pp. 453-476.

Breiger, R.L., 2000. A tool kit for practice theory. Poetics 27, 91-115.

Breiger, R.L., Boorman, S.A., Arabie, P., 1975. An algorithm for clustering relational data, with applications to social network analysis and comparison with multidimensional scaling. Journal of Mathematical Psychology $12,328-383$.

Cao, Z., Kim, K.H., Roush, F., 1984. Incline Algebra and its Applications. Ellis Horwood, New York.

Chubb, C., 1986. Collapsing binary data for algebraic multidimensional representation. Journal of Mathematical Psychology 30, 161-187.

Coombs, C.H., 1964. A Theory of Data. Wiley, New York.

De Boeck, P., Rosenberg, S., 1988. Hierarchical classes: model and data analysis. Psychometrika 53, 361-381.

Doignon, J.-P., Ducamp, A., Falmagne, J.-C., 1984. On realizable biorders and the biorder dimension of a relation. Journal of Mathematical Psychology 28, 73-109.

Doignon, J.-P., Falmagne, J.-C., 1984. Matching relations and the dimensional structure of social choices. Mathematical Social Sciences 7, 211-229.

Doignon, J.-P., Falmagne, J.-C., 1985. Spaces for the assessment of knowledge. International Journal of Man-Machine Studies 23, 175-196.

Eckart, C., Young, G., 1936. The approximation of one matrix by another of lower rank. Psychometrika 1, 211-218.

Fararo, T.J., Doreian, P., 1984. Tripartite structural analysis. Social Networks 6, 141-175.

Feick, L.F., 1987. Latent class models for the analysis of behavioral hierarchies. Journal of Marketing Research 24, 174-187.

Foster, B.L., Seidman, S.B., 1984. Overlap structures of ceremonial events in two Thai villages. Thai Journal of Development Administration 24, 143-157.

Freeman, L.C., 1980. $Q$-analysis and the structure of friendship networks. International Journal of Man-Machine Studies 12, 367-378.

Freeman, L.C., 1992. La resurrection des cliques: application du Treillis de Galois. Bulletin de Méthodologie Sociologique 37, 3-24.

Freeman, L.C., 1996. Cliques, Galois lattices, and the structure of human social groups. Social Networks 18 , $173-187$.

Freeman, L.C., White, D.R., 1993. Using Galois lattices to represent network data. In: Marsden, P.V. (Ed.), Sociological Methodology. American Sociological Association, Washington, DC, 1993, pp. 127-146.

Ganter, B., Wille, R., 1989. Conceptual scaling. In: Roberts, F. (Ed.), Applications of Combinatorics and Graph Theory in the Biological and Social Sciences. Springer, New York, pp. 139-167.

Ganter, B., Wille, R., 1999. Formal Concept Analysis: Mathematical Foundations. Springer, Berlin.

Kim, K.H., 1982. Boolean Matrix Theory and Applications. Dekker, New York.

Martin, J.L., Wiley, J., 1999. Algebraic representations of beliefs and attitudes: partial order models for item responses. Sociological Methodology 29, 113-146.

Martin, J.L., Wiley, J., 2000. Algebraic representations of beliefs and attitudes. II. Microbelief models for dichotomous belief data. Sociological Methodology 30, 123-164.

Mickey, M.R., Mundle, P., Engelman, L., 1983. Boolean factor analysis. In: Dixon, W.J. (Ed.), BMDP Statistical Software. University of California Press, Berkeley, CA, pp. 538-545.

Mirkin, B.G., 1987. Additive clustering and qualitative factor analysis methods for similarity matrices. Journal of Classification 4, 7-31.

Mische, A., Pattison, P.E., 2000. Composing a civic arena: publics, projects, and social settings. Poetics 27, 163-194.

Pattison, P.E., 1993. Algebraic Models for Social Networks. Cambridge University Press, New York.

Pattison, P.E., Bartlett, W.K., 1982. A factorization procedure for finite algebras. Journal of Mathematical Psychology 25, 51-81. 
Pattison, P.E., Robins, G.L., 2000. Neighborhood-based models for social networks. Presentation at the International Conference on Social Networks, Vancouver, 12-16 April 2000.

Pattison, P.E., Robins, G.L., in press. Neighborhood-based models for social networks. Sociological Methodology. Rindskopf, D., 1983. A general framework for using latent class analysis to test hierarchical and non-hierarchical learning models. Psychometrika 48, 85-97.

Sanchez, E., 1976. Resolution of composite fuzzy relation equations. Information and Control 30, 38-48.

Schönemann, P.H., 1970. On metric multidimensional unfolding. Psychometrika 35, 167-176.

Skvoretz, J., Faust, K., 1999. Logit models for affiliation networks. Sociological Methodology 29, 253-280.

White, D.R., Duquenne, V., 1996. Social networks and discrete structure analysis. Social Networks, 18, 169-318 (special issue).

White, H.C., Boorman, S.A., Breiger, R.L., 1976. Social structure from multiple networks. I. Blockmodels of roles and positions. American Journal of Sociology 81, 730-780.

Wilson, T.P., 1982. Relational networks: an extension of sociometric concepts. Social Networks 4, $105-116$.

Wolff, K.E., Gabler, S., 1998. Comparison of visualizations in formal concept analysis and correspondence analysis. In: Blasius, J., Greenacre, M. (Eds.), Visualization of Categorical Data. Academic Press, San Diego, pp. 85-97.

Young, G., Householder, A.S., 1938. Discussion of a set of points in terms of their mutual distances. Psychometrika $3,19-22$ 